\title{
The Effect of Nd:YAG Iridotomy on Intraocular Pressure in Hypertensive Eyes with Shallow Anterior Chambers
}

\author{
J. N. McGALLIARD and P. K. WISHART \\ Liverpool
}

\begin{abstract}
Summary
Eighty one eyes of 48 patients with raised IOP and shallow anterior chambers underwent Nd:YAG iridotomy to prevent further angle closure glaucoma and to remove any pupil block that might be contributing to the raised IOP. These eyes were followed for 18-34 months to assess the effect on IOP control.

Seventy three eyes of $\mathbf{4 3}$ patients were asymptomatic. In eyes with no PAS none responded with drop in IOP but in eyes with well established PAS 69\% showed a drop in IOP.

By comparison eight eyes of five patients had a history of episodes of subacute angle closure glaucoma. All had PAS present pre-operatively. Iridotomy reduced IOP in $87.5 \%$ of the eyes in this group and rendered $100 \%$ symptom free.

Permanent posterior synechiae between lens and iris were an invariable complication in those eyes that required long-term miotic treatment for IOP control following iridotomy.

In asymptomatic eyes with chronic pressure elevation and narrow angles laser iridotomy is unlikely significantly to reduce the IOP. We recommend that in such eyes iridotomy should be reserved for those eyes with established closure of the angle by PAS in which IOP control can be achieved without the use of miotics.
\end{abstract}

A shallow anterior chamber is regarded as a sign of considerable significance in an eye with raised intraocular pressure (IOP). ${ }^{1}$ Its recognition immediately prompts the question as to whether or not the raised IOP is a consequence of relative pupil block. Such eyes are also at risk of developing acute angle closure glaucoma which carries the risk of sudden and severe visual loss. ${ }^{2}$

It is the danger of angle closure glaucoma which correctly induces caution in the management of eyes with shallow anterior chambers. The clinician must use his judge- ment to determine the relative risk of angle closure glaucoma in each eye and treat accordingly.

Eyes with a history of acute or subacute angle closure glaucoma require peripheral iridectomy or laser iridotomy. ${ }^{3}$ There exists however, a large group of patients with no history of acute or subacute attacks of angle closure glaucoma who have raised IOP, shallow anterior chamber and narrow angles. It has been suggested ${ }^{4,5}$ that such eyes should undergo surgical iridectomy or laser iridotomy to relieve any relative degree of pupil 
block thereby lowering the IOP and removing the risk of developing angle closure glaucoma in the future.

The aim of this study was to determine the benefit of $\mathrm{Nd}$ :YAG laser iridotomy to such asymptomatic eyes with shallow anterior chambers and raisd IOP. As a control group, eyes with a history of subacute angle closure glaucoma presenting during the study period underwent $\mathrm{Nd}$ :YAG iridotomy and were followed concurrently with the study group.

\section{Materials and Methods}

Eighty one hypertensive eyes (48 patients) in which Nd:YAG iridotomy was clinically indicated were followed to determine the long term effects of laser iridotomy on IOP and anterior chamber angle appearance and to identify any long-term deleterious effect of treatment. The indications for laser iridotomy were:-

(1) Symptoms and/or signs of subacute angle closure glaucoma.

(2) A diagnosis of chronic angle closure glaucoma as shown by peripheral anterior synechiae (PAS) in the superior angle with visual field loss and/or glaucomatous disc damage.

(3) Hypertensive eyes with PAS in the superior angle without detectable glaucomatous damage to disc or field.

(4) Hypertensive eyes without PAS but in which a degree of peripheral iris bombe was thought to be a contributory factor to the raised IOP.

Forty three patients, in whom 73 iridotomies were performed were asymptomatic when the elevated IOP was diagnosed. The remaining five patients in whom eight iridotomies were performed, gave a history suggestive of subacute angle closure attacks.

For inclusion in the study all eyes presented with intraocular pressures greater than 22 $\mathrm{mmHg}$ and an axial anterior chamber depth of less than $2.3 \mathrm{~mm}$. Any eye with a history of iritis or iris anomalies was excluded.

All patients were assessed initially by one of us (PKW) by slit-lamp examination including Goldmann applanation tonometry, axial anterior chamber depth estimation by HaagStreit pachymmetry, indentation gonioscopy using a Zeiss four mirror gonioscope and bio- microscopic disc assessment. Visual field assessment by either the Hensen or automated Humphrey field analysers was performed where appropriate.

Iridotomy was performed using either the Coopervision or Sharplan Nd:YAG laser by the following technique. If present, a peripheral iris crypt in the upper quadrant of the iris was selected. With a moderate power setting 2.5-5 mJ a full thickness iridotomy was often achieved with a single shot. If not full thickness, or if small, this iridotomy was then enlarged using further low energy (1-2 mJ) shots. Intraocular pressures were checked between one and two hours following iridotomy and acetazolamide and short-term (less than 24 hours) pilocarpine $2 \%$ prescribed if indicated.

Post-operatively the treated eyes received prednisolone phosphate $0.5 \%$ drops four times a day for a week. All patients were assessed at regular intervals by applanation tonometry, anterior chamber depth measurement and indentation gonioscopy. No reduction in anti-glaucoma medications was made until the second clinic visit follow iridotomy.

All patients have completed a minimum of eighteen months follow-up since iridotomy (range 18-34 months).

\section{Results}

Forty eight patients were identified during the study period. The axial anterior chamber depths are shown in Figure 1 with a peak incidence between 1.9 and $2.0 \mathrm{~mm}$.

\section{Symptomatic Patients}

Five patients presented with symptoms suggestive of previous attacks of subacute angle closure glaucoma. They comprised two males and three females. The average age at presentation was 63 years (range 43-69 years) with an average anterior chamber depth of 1.96 $\mathrm{mm}$ (range 1.5-2.2 mm). Average IOP on presentation was $33.0 \mathrm{mmHg}$ (range 23-50 $\mathrm{mmHg}$ ).

In this group eight iridotomies were performed. In seven $(87.5 \%)$ the intraocular pressure was reduced following iridotomy. One of these seven eyes was normotensive without treatment following iridotomy. The average drop in IOP was $9.2 \mathrm{mmHg}$ (range 
Anterior chamber depth profile

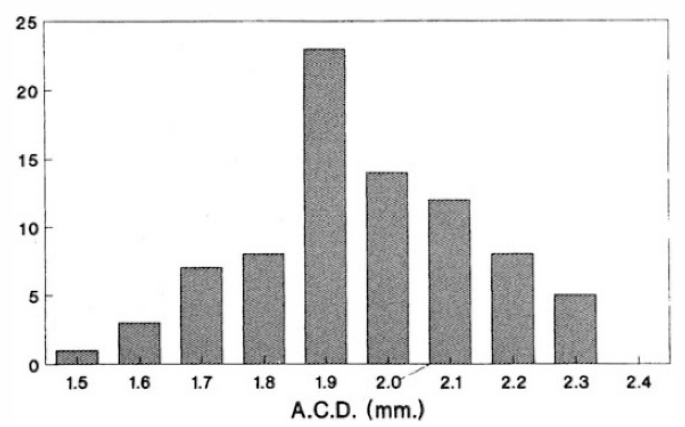

Fig. 1. Distribution of anterior chamber depth in all treated eyes.

4-24 mmHg). All eight eyes in this group had peripheral anterior synechiae present in the superior angle and in three eyes these were more extensive than three clock hours. $62.5 \%$ of the eight eyes had evidence of glaucomatous damage either of the optic disc or the visual field before iridotomy.

Following iridotomy no further episodes of subacute angle closure occurred in any patient. There was no change in axial anterior chamber depth following iridotomy. Repeated gonioscopy following iridotomy showed no progression in peripheral anterior synechiae.

\section{Asymptomatic Patients}

Seventy three eyes of 43 patients were treated. They comprised fourteen males and twenty nine females. The average age at presentation was 70.2 years (range $45-87$ years) with an average anterior chamber depth of $2.02 \mathrm{~mm}$ (range 1.6-2.3 $\mathrm{mm}$ ). The average IOP on presentation was $27.9 \mathrm{mmHg}$ (range 23-44 mmHg).

In thirty eight patients (64 eyes) there was no reduction in IOP following iridotomy. In the remaining five patients, nine eyes underwent iridotomy and this resulted in an average drop in IOP of $7.3 \mathrm{mmHg}$ (range 4-18 $\mathrm{mmHg}$ ). Of these nine eyes only one became normotensive without treatment.

Comparison of those eyes which responded with a drop in IOP to those showing no response demonstrated no significant difference in pre-operative IOP, axial anterior chamber depth or age of patients. Glaucomatous damage of either the optic disc or visual field was present in $32.8 \%$ of those eyes which showed no change in IOP following iridotomy and in $22 \%$ of those eyes which responded with a drop in IOP. This difference is not statistically significant $(p=0.46)$. The extent of visual field loss in these asymptomatic eyes is shown in Table I.

The presence of PAS was however significantly more common in the group which responded to iridotomy. One hundred per cent of the nine eyes with reduced IOP had established PAS in the superior angle of at least two clock hours in extent. Of the 64 eyes in which iridotomy had no pressure lowering effect, PAS were present in $42.2 \%$ but in most cases these were only fine bridging strands rather than well established contiguous bands of PAS (Table II).

The complications of iridotomy encountered in this series are shown in Table III. In the early phase, hyphaema was noted in $27.1 \%$ of cases. This was microscopic in all but one case which developed a blood level visible $1 \mathrm{~mm}$ above the limbus. In two eyes iridotomy was unsuccessful at the first sitting and required further treatment the following day. Elevation of IOP of $5 \mathrm{mmHg}$ or greater following iridotomy was recorded in $38.3 \%$ of cases. In three eyes the hypertensive effect of iridotomy was greater than $20 \mathrm{mmHg}$ and these patients were admitted to hospital for stabilisation. None suffered a drop in visual acuity as a consequence of the pressure peak.

The complication of iridotomy which was of most clinical importance was the development of posterior synechiae. In patients requiring long-term post-operative miotics for control of the IOP, all such eyes developed dense posterior synechiae which were resistant to intensive mydriasis (Fig. 2). Use of pilocarpine to control the immediate post-laser pressure rise did not cause permanent posterior synechiae, but when reintroduced for more than immediate control, synechiae were the inevitable consequence. These synechiae occurred even when miotics were reintroduced after an interval of more than two weeks post iridotomy.

In three eyes cataract extraction became necessary during the follow-up period. In these eyes significant lens opacity was present before iridotomy was performed. 
Table I Extent of visual field loss in asymptomatic patients ( 73 eyes)

\begin{tabular}{lcc}
\hline & $\begin{array}{c}\text { No change } \\
\text { in IOP }\end{array}$ & $\begin{array}{c}\text { Fall in } \\
\text { IOP }\end{array}$ \\
\hline None & 43 & 7 \\
Arcuate area only & 14 & 2 \\
Break through in periphery & 4 & 0 \\
Loss of central fixation & 3 & 0 \\
\hline
\end{tabular}

\section{Discussion}

The aim of this study was to assess the effect of laser iridotomy on a group of asymptomatic eyes with raised IOP and shallow anterior chamber. This combination of features is an increasingly common problem when dealing with an elderly population since anterior chamber depth decreases with age and the incidence of glaucoma increases with age. ${ }^{6}$

A relative degree of pupil block in an eye with a shallow AC is widely believed to be one factor contributing to IOP elevation in hypertensive eyes with shallow anterior chambers and narrow angles. ${ }^{7}$ Medical treatment of such eyes may worsen the situation, as pilocarpine has been shown to further shallow the peripheral $\mathrm{AC}^{8}$ in those $50 \%$ of eyes in which it causes a shallowing of the axial AC. This effect may further predispose the eye to develop PAS of the superior angle or develop acute ACG. Sympathomimetics may cause crowding of an already narrow angle especially if used in conjunction with beta blockade which renders the alpha receptors hypersensitive to catecholamines thereby increasing the risk of development of acute angle closure glaucoma. ${ }^{9}$

Many studies have been published attesting to the beneficial effect of iridectomy/otomy in hypertensive eyes and narrow angles after relief of pupil block. Pollack ${ }^{5}$ reported the successful treatment of eyes with narrow angles and glaucoma by surgical iridectomy and concluded that the existence of combined

Table II Extent of PAS in asymptomatic patients (73 eyes)

\begin{tabular}{lccc}
\hline & Total & \multicolumn{2}{c}{ Fall in IOP } \\
\hline No PAS & 37 & 0 & $0 \%$ \\
Fine strands & 23 & 0 & $0 \%$ \\
Established PAS & 13 & 9 & $69 \%$ \\
\hline
\end{tabular}

mechanism glaucoma was extremely unlikely and that glaucoma in the presence of a narrow angle was likely to represent chronic angle closure glaucoma. A beneficial effect of surgical iridectomy in eyes with chronic angle closure glaucoma has been reported by Lowe, ${ }^{10}$ Watson and Playfair, ${ }^{11}$ Gelber and Anderson, ${ }^{12}$ and Murphy and Spaeth. ${ }^{13}$ This last study also reported iridectomy to be a successful treatment in $50 \%$ of cases with chronic open angle glaucoma that had a superimposed angle closure attack. Coakes et al. ${ }^{14}$ showed the deepening of the peripheral AC that follows iridectomy and Hitchings and LloydJones ${ }^{15}$ showed a beneficial effect of this treatment in eyes with chronic simple glaucoma and narrow angles.

A similar wealth of literature supports laser iridotomy as the treatment of choice in eyes with chronic angle closure glaucoma. Quigley ${ }^{16}$ describes Argon laser iridotomy as having particular advantages in the treatment of chronic angle closure glaucoma. Ritch ${ }^{17}$ and later Gieser and Wilensky ${ }^{18}$ have advocated laser iridotomy as the procedure of choice in chronic angle closure glaucoma. The relative ease of performance of laser iridotomy probably helps explain the fourfold increase in the rate of iridotomies over surgical iridectomies reported by Rivera et al. ${ }^{19}$ in the first year in which Argon laser iridotomy became an accepted method of treatment at the Bascom Palmer Eye Institute, Miami. However Wishart et al. ${ }^{20}$ reported no benefit of laser iridotomy or surgical iridectomy in a group of eyes with chronic angle closure glaucoma and significant disc damage.

In this study the symptomatic patients were recruited as a comparison and to ensure that our methodology was correct. Iridotomy in this group with symptoms of subacute angle closure glaucoma and PAS in the superior angle was $100 \%$ successful in controlling the

Table III Complications of iridotomy (81 eyes)

\section{Early}

Hyphaema $\ldots \ldots \ldots \ldots \ldots \ldots \ldots \ldots \ldots \ldots \ldots 22$

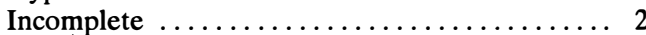

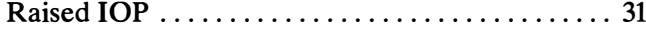

Late

Closure ...................... 1

Posterior synechiae $\ldots \ldots \ldots \ldots \ldots \ldots \ldots \ldots 17$ 


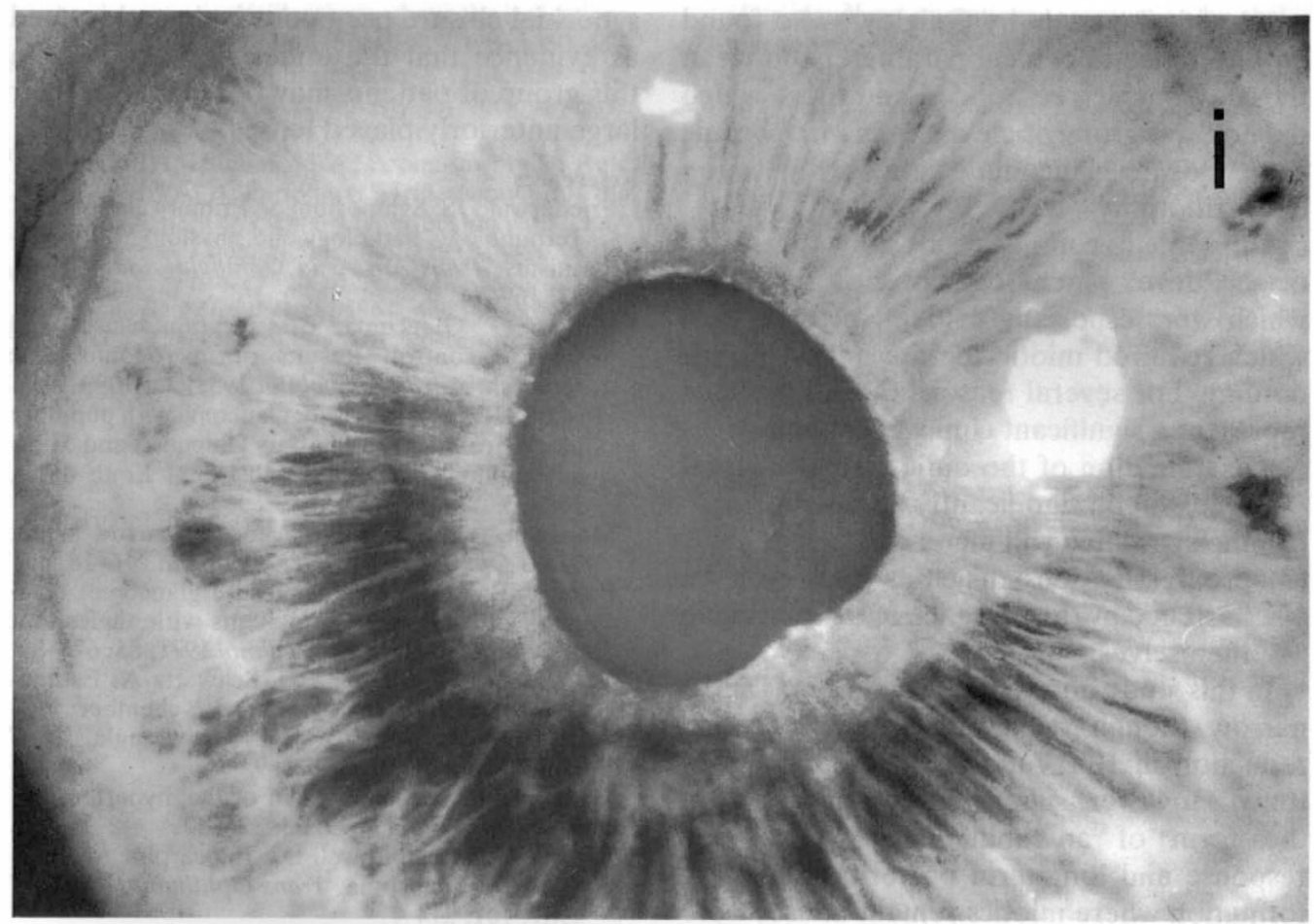

Fig. 2. Slit-lamp photograph showing extensive posterior synechiae preventing effective mydriasis in eye following Nd:YAG iridotomy (I) and long-term Pilocarpine.

symptoms associated with angle closure attacks and in $87.5 \%$ resulted in reduction of the intraocular pressure.

In the asymptomatic patients iridotomy resulted in a drop of IOP in only $12.3 \%$ of eyes. All of these $12.3 \%$ had significant PAS pre-operatively. From this result it can be assumed that pupil block was responsible for some of the elevation of IOP in this subgroup. In only one eye $(1.4 \%)$ was the post iridotomy IOP within the normal range without treatment. The cause of the residual hypertension in the other eight is not known but may represent either a pre-existing outflow insufficiency or a secondary trabecular damage due to prolonged appositional closure. In the remaining $87.7 \%$ of asymptomatic eyes laser iridotomy had no pressure lowering effect. Included in this $87.7 \%$ are all those eyes in which the angle was free of PAS. This result does not support the findings of Romano et al. ${ }^{21}$ who reported a more marked hypotensive response in hypertensive eyes without PAS following Nd:YAG Iridotomy. Our study has demonstrated a clear distinction in the response to treatment between those eyes with established PAS (69\% fall in IOP) and those with no PAS or early fine bridging strands (both latter groups $0 \%$ fall in IOP) (Table II). These results indicate to us that established contiguous bands of PAS represent a different entity to fine isolated bridging strands. Whereas established PAS are likely to represent an angle closure mechanism and will progress to chronic angle closure glaucoma $^{22}$ if pupil block is not relieved, isolated bridging strands may be an incidental finding in an eye with narrow angles and should be observed rather than be regarded as indicative of an angle closure mechanism. If these are seen to progress on follow-up gonioscopy then intervention is indicated, but if static, then pupil block is not responsible for their presence.

Glaucomatous damage of the optic disc or visual field in combination with PAS was not of any predictive value in showing which eyes would respond to iridotomy. This is in agree- 
ment with the study by Quigley ${ }^{16}$ who found no difference in clinical parameters between those eyes which responded and those which did not, in a group of eleven eyes with chronic angle closure glaucoma treatment by Argon laser iridotomy.

Our table of complications show the extent of the development of posterior synechiae which were especially evident in those eyes which required miotic therapy following iridotomy. For several reasons these synechiae represent a significant clinical problem:-

(1) examination of the optic disc and visual field will be significantly hampered,

(2) the synechiae will increase

(3) progressive lens opacities may even be hastened by the presence of the synechiae themselves. ${ }^{23}$

In this study no eye with shallow $\mathrm{AC}$ and a narrow though open angle experienced a reduction in IOP following Nd:YAG iridotomy. However, significant complications in the form of an immediate hypertensive response and long-term posterior synechiae formation where miotics remain in use, were observed. For these reasons we feel that in asymptomatic hypertensive eyes in the absence of PAS Nd:YAG laser iridotomy is not indicated, no matter how shallow the anterior chamber.

The presence of PAS in the superior angle of a hypertensive eye with a shallow $\mathrm{AC}$ and a narrow angle indicates an angle closure mechanism. Iridotomy will prevent further development of synechial closure and reduce the risk of an attack of acute angle glaucoma, but as mentioned the price for these benefits for those eyes requiring miotics is the development of posterior synechiae. If the IOP can be controlled without the use of miotics then Nd:YAG iridotomy is indicated. Only one asymptomatic eye $(1.4 \%)$ was rendered treatment free by Nd:YAG iridotomy and therefore in those eyes requiring miotics it may be better to perform filtering surgery or in the presence of opacities lens extraction to reduce IOP thus avoiding miotic induced posterior synechiae.

Two studies recently ${ }^{24,25}$ reported the beneficial effect of lens extraction on IOP control in chronic angle closure glaucoma. This response was independent of the degree of synechial closure pre-operatively and is cited as evidence that the underlying problem in this group of patients may be related to their large anteriorly placed lenses.

\section{References}

${ }^{1}$ Friederwald JS: Symposium on Primary glaucoma: I Terminology, pathology and physiological mechanisms. Trans Am Acad Ophthalmol Otol 1949, 53: $169-74$.

${ }^{2}$ Barkan O: Glaucoma; Classification causes and surgical control. Results of microgonioscopic research. Am J Ophthalmol 1938, 22: 1099-113.

${ }^{3}$ Hoskins HD: Angle closure glaucoma with pupillary block. In: Becker-Shaffers Diagnosis and Therapy of the Glaucomas. Mosby. St Louis 1989, 208-33.

${ }^{4}$ Mapstone R: Clinical significance of narrow angle. Trans Ophthalmol Soc UK 1978, 98: 216-18.

${ }^{5}$ Pollack IP: Chronic angle-closure glaucoma: diagnosis and treatment in patients with angles that appear open. Arch Ophthalmol 1971, 85: 676-89.

${ }^{6}$ Van Herrick W, Shaffer RN, Schwartz A: Estimation of width of angle of anterior chamber: incidence and significance of a narrow angle. $A m \mathrm{~J}$ Ophthalmol 1969, 68: 626-9.

${ }^{7}$ Mapstone R: Mechanisms in ocular hypertension. Br J Ophthalmol 1979, 63: 325-30.

${ }^{8}$ Hitchings RA and Powell DJ: Pilocarpine and narrow-angle glaucoma. Trans Ophthalmol Soc UK 1981, 101: 214-7.

${ }^{9}$ James I: The pharmacological effect of the betablocking agents used in the management of glaucoma. Symposium: New Vistas in the Detection and Management of Glaucoma. Brussels, September 1987.

${ }^{10}$ Lowe R: The natural history and treatment of primary angle-closure glaucoma. Am J Ophthalmol 1966, 65: 624-51.

${ }^{11}$ Playfair TM and Watson PG: Management of chronic or intermittent primary angle-closure glaucoma: a long-term follow-up of the results of peripheral iridectomy used as an initial procedure. Br J Ophthalmol 1979, 63: 23-8.

${ }^{12}$ Gelber EC and Anderson DR: Surgical decisions in chronic angle-closure glaucoma. Arch Ophthalmol 1976, 94: 1481-4.

${ }^{13}$ Murphy MB and Spaeth GL: Iridectomy in primary angle-closure glaucoma. Arch Ophthalmol 1974, 91: 114-22.

${ }^{14}$ Coakes RL, Lloyd-Jones D, Hitchings RA: Anterior chamber volume. Its measurement and clinical application. Trans Ophthalmol Soc UK 1979, 99: 78-81.

${ }^{15}$ Hitchings RA and Lloyd-Jones D: Peripheral iridectomy for chronic simple glaucoma with narrow angles. Trans Ophthalmol Soc UK 1979, 99: 84-8.

${ }^{16}$ Quigley HA: Long-term follow-up of laser iridotomy. Ophthalmology 1981, 88: 218-24.

${ }^{17}$ Rich R: The treatment of chronic angle-closure glaucoma. Ann Ophthalmol 1981, 13: 12-13.

${ }^{18}$ Gieser DK and Wilensky JT: Laser iridectomy in the management of chronic angle-closure glaucoma. Am J Ophthalmol 1984, 98: 446-50.

${ }^{19}$ Rivera AH, Brown RH, Anderson DR: Laser irido- 
tomy vs surgical iridectomy-have the indications changed? Arch Ophthalmol 1985, 103: 1350-4.

${ }^{20}$ Wishart PK, Nagasubramanian S, Hitching RA: Argon Laser Trabeculoplasty in Narrow Angle Glaucoma. Eye 1987, 1: 567-76.

${ }^{21}$ Romano JH, Hitchings RA, Pooinasawmy D: Role of Nd:YAG Peripheral Iridectomy in the Management of Ocular Hypertension with a narrow angle. Ophthal Surg 1988, 19: 814-16.

${ }^{22}$ Lowe RF: Primary creeping angle-closure glaucoma. Br J Ophthalmol 1964, 48: 544-50.

${ }^{23}$ Philip CI, Clark CV, Levy AM: Posterior synechiae after glaucoma operations: aggravation by shallow anterior chamber and pilocarpine. $\mathrm{BrJO} \mathrm{Oph}$ thalmol 1987, 71: 428-32.

${ }^{24}$ Wishart PK and Atkinson PL: Extracapsular Cataract Extraction and Posterior Chamber Lens Implantation in patients with primary chronic angle-closure glaucoma. Effect on IOP Control. Eye 1989, 3: 706-12.

${ }^{25}$ Greve EC: Primary Angle Closure Glaucoma. Extracapsular Cataract Extraction or Filtering Procedure? Internat Ophthalmol 1988, 12: $157-62$. 